\title{
Design Hybrid Evolutionary PSO Aiding Miniature Aerial Vehicle Controllers
}

\author{
Juing-Shian Chiou, ${ }^{1}$ Huu-Khoa Tran, ${ }^{2}$ and Shou-Tao Peng ${ }^{2}$ \\ ${ }^{1}$ Department of Electrical Engineering, Southern Taiwan University of Science and Technology, Tainan City, Taiwan \\ ${ }^{2}$ Mechanical Engineering Department, Southern Taiwan University of Science and Technology, Tainan City, Taiwan
}

Correspondence should be addressed to Juing-Shian Chiou; jschiou@mail.stust.edu.tw

Received 22 August 2014; Accepted 30 September 2014

Academic Editor: Stephen D. Prior

Copyright (C) 2015 Juing-Shian Chiou et al. This is an open access article distributed under the Creative Commons Attribution License, which permits unrestricted use, distribution, and reproduction in any medium, provided the original work is properly cited.

Based on some sort of simplified fuzzy reasoning methods and PID parameters, many fuzzy-PID controller schemes are applied to control the complicated systems, recently. Mathematical optimization calculations are making in encouraging EP with PSO yielding a higher-quality solution. In this paper, a novel HEPSO algorithm as an improved variant of stochastic optimization strategy PSO, which assigns optimization to fuzzy-PID control gains, is established. The benefit integration design of the HEPSO algorithm structure is generating and updating the new parameters for the fuzzy-PID control schemes in the short setting and operation time. The proposed controllers have demonstrated performance to MAV models.

\section{Introduction}

The production systems are difficult in representing their actual behavior as well as in optimizing and planning their performance. For this reason, simulation modeling methods have been used extensively to mimic the behaviors and to provide a flexible platform for optimal parameter design and system improvement. Various optimization methods, based on soft computing, for instance, evolutionary algorithm, swarm intelligence, and ant colony optimization, have been explored and applied to solve the problem in consideration of criterion and attained the sensible results.

Introduced by Lawrence J. Fogel in the USA for the first time, 1966 [1], evolutionary programming (EP), with continuing development by his son D. B. Fogel, is parallel to global search techniques that share the concepts of natural genetics and evolution theory. Similarly to genetic programming, which allows evolving numerical parameters, however, the EP program optimization structure is fixed. Its main variation operator is mutation and selection strategy whereas neglect the crossover operation.

Inspired by social behavior of bird flocks and fish swarms, particle swarm optimization (PSO) was developed by Kennedy and Eberhart [2] in 1995. PSO is a swarm intelligence technique, founded on stochastic and population-based adaptive optimization. The primary PSO is effortless with simply a few adjusted parameters; however, when comparing with other methods such as neural network, machine learning, and genetic computation, the better performances in computing speed, accuracy, and small memory size are achieved.

Different hybrid PSOs have been proposed due to computing optimal objective function, recently. HGAPSO, which is suggested by Juang [3], incorporates GA's evolutionary operations of elite strategy, where the elites are stored in upper-half of the best-performing individuals in a population. For estimating parameters of nonlinear systems, an effective hybrid optimization strategy by incorporating the jumping property of simulated annealing (SA) into PSO, namely, PSOSA, was presented by $\mathrm{Li}$ et al. [4]. A parallel algorithm of PSO and differential evolution (DE) executed in parallel to enhance the population with frequent information sharing was demonstrated by Niu and Li [5]. The combination of PSO and gravitational search algorithm (GSA) was introduced by Mirjalili and Hashim based on gravitation force [6]. A novel hybrid binary particle swarm optimization algorithm HBPSO by combination of the PSO's concept and GA was presented by Menhas et al. [7]. Cuckoo search (CS) 
and particle swarm optimization (PSO), inspired by nature metaheuristic algorithm for solving optimization problems, were established by Ghodrati and Lotfi [8]. HPSOM, to integrate the PSO with genetic algorithm mutation method, was introduced by Esmin and Matwin [9].

Evolutionary programming (EP) algorithm, which only has a mutation operator and an individual selection strategy, neglects a crossover operator. All of the revealed methods above can increase the convergence speed. This research paper exploits the HEPSO optimization algorithm and implements it to MAV models.

Because of the simple control structure, low cost, and ease to design, conventional PID controllers are applied to many industrial fields. Moreover, due to regulated control gains, a new hybrid control approach fuzzy-PID is applied for an autonomous minihelicopter [10]. Since information about the physical process is vague and data available is scarce, fuzzy rule-based model is suitable. The fuzzy logic controller (FLC) has been proved to be one of the most efficient and systematic approaches to deal with such kinds of problems in the fact that its control capability arises from emulating human logic instead of accurate mathematical model [11]. One drawback of designed fuzzy system is that the extracted rules are independent of the membership functions, especially for a complex system problem with a large number of input variables. Therefore no guarantee that the fuzzy system obtained will have sufficiently good performance. In many cases, the system's performance, however, can be improved by further tuning the membership functions and selecting suitable fuzzification and defuzzification methods [10, 12-14].

This paper deals with the design of triangular membership functions of fuzzy-PID controller. The parameter gains are then evaluated on the HEPSO by using fitness functions in association with the system's performance indicesintegral of time multiplied by absolute error (ITAE) [15]. The controller's designs assign a task to MAV models: rotor speed, servo motor position, and altitude control to show the benefits in enhancing performances.

\section{Fuzzy-PID Controller Description}

PID controllers' main functions are improving the dynamic response and reducing or eliminating the steady-state errors. In this paper, an improvement to such widely used fuzzy-PID controllers by incorporating a fast learning PID controller with parameters yielding a high-quality solution is proposed.

The tracking error $e(t)$ and the differential tracking error $d e(t)$, as shown in Figure 1, are the inputs of the fuzzy inference system [16]. The formal triangular membership functions are used for the two inputs $e(t)$ and $d e(t)$ and the output $C I$.

The dynamic behavior of fuzzy logic controllers (FLC) is described by a set of linguistic rules, based on expert knowledge. Mandani's MIN-MAX inference engine type and center of area method (COA) defuzzification are employed in this paper. The sliding range of ZE limited the error range and the optimal system condition regulated the PID controller.

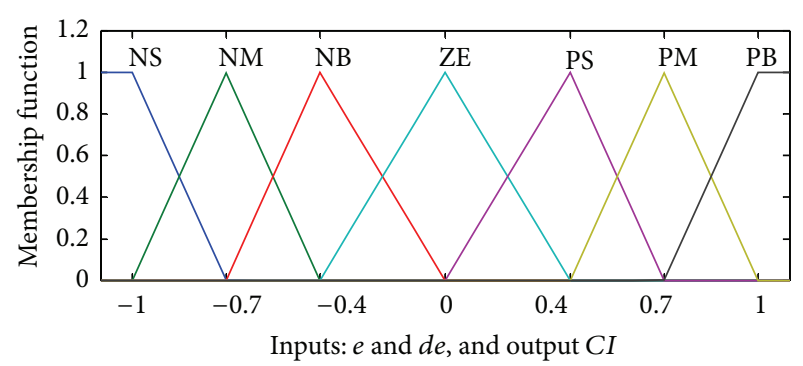

Figure 1: Inputs $e$ and $d e$ and output $C I$ of fuzzification interface.

TABLE 1: The fuzzy rule table.

\begin{tabular}{|c|c|c|c|c|c|c|c|c|}
\hline \multirow{2}{*}{\multicolumn{2}{|c|}{$C I(t)$}} & \multicolumn{7}{|c|}{$e(t)$} \\
\hline & & NB & NM & NS & ZE & PS & PM & $\mathrm{PB}$ \\
\hline \multirow{7}{*}{$d e(t)$} & NB & ZE & NS & $\mathrm{NM}$ & $\mathrm{NM}$ & NB & NB & $\mathrm{NB}$ \\
\hline & NM & PS & $\mathrm{ZE}$ & NS & NM & NM & NB & NB \\
\hline & NS & PM & PS & ZE & NS & NM & NM & NB \\
\hline & $\mathrm{ZE}$ & PM & PM & PS & $\mathrm{ZE}$ & NS & NM & NM \\
\hline & PS & РB & $\mathrm{PM}$ & PM & PS & $\mathrm{ZE}$ & NS & NM \\
\hline & $\mathrm{PM}$ & РB & $\mathrm{PB}$ & PM & PM & PS & $\mathrm{ZE}$ & NS \\
\hline & $\mathrm{PB}$ & $\mathrm{PB}$ & $\mathrm{PB}$ & $\mathrm{PB}$ & PM & $\mathrm{PM}$ & PS & $\mathrm{ZE}$ \\
\hline
\end{tabular}

Since its combination yields the basic implementation parameters of the fuzzy control algorithm, seven partitions are decomposed: negative big (NB), negative medium (NM), negative small (NS), zero (ZE), positive small (PS), positive medium (PM), and positive big (PB) partitions. The fuzzy control rules are then designed as shown in Table 1.

Most physical empirical systems have inherently intractable characteristics such as high order and nonlinearity. Thus, the satisfied performance requirement obtained by the fuzzy-PID controllers has been tackled in many studies $[10,12,13,17,18]$. In a fuzzy-PID structure, the endeavor controller can be written as

$$
u=C I+u_{\mathrm{PID}}=C I+k_{P} \times e+k_{I} \int e+k_{D} \times \frac{d e}{d t} .
$$

\section{Hybrid Evolutionary PSO}

The primary inspiration of fundamental PSO algorithm that a great number of particles move around in a multidimensional space was the social behavior of animals, such as the schooling of fish, the flocking of birds, and the swarm theory [2]. The fundamental PSO algorithm can be verified by [2$9,11,19,20]$

$$
\begin{aligned}
v_{i D}(t+1)= & \omega \times v_{i D}(t)+c_{1} \times \operatorname{rand}_{1}(\cdot) \times\left(p_{i D}(t)-x_{i D}(t)\right) \\
& +c_{2} \times \operatorname{rand}_{2}(\cdot) \times\left(p_{g D}(t)-x_{i D}(t)\right) \\
& x_{i D}(t+1)=x_{i D}(t)+v_{i D}(t+1),
\end{aligned}
$$

where $v_{i D}$ is the velocity of particle $i$ and $x_{i D}$ is the position of particle $i$ with objective value fitness and $p_{i D}$ is the best historical position of particle $i$ itself, while $p_{g D}$ is the global best position. The inertia weight $\omega$ is a user-defined that 


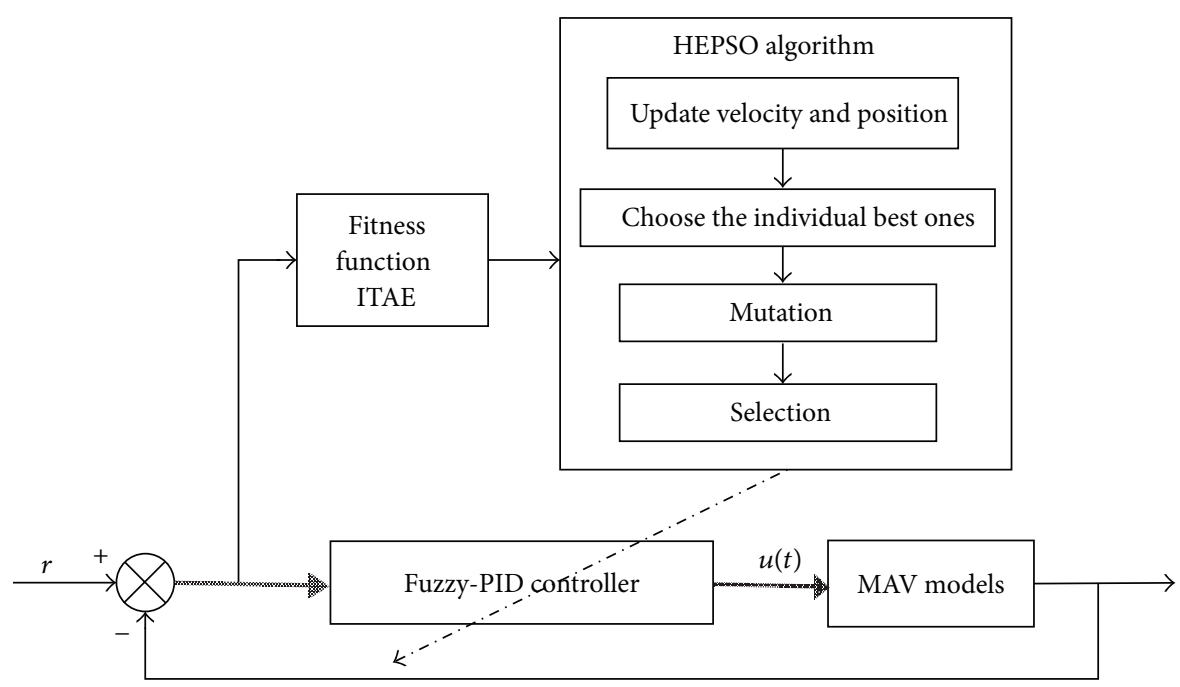

FIgURE 2: The MAV controllers were aided by HEPSO algorithm.
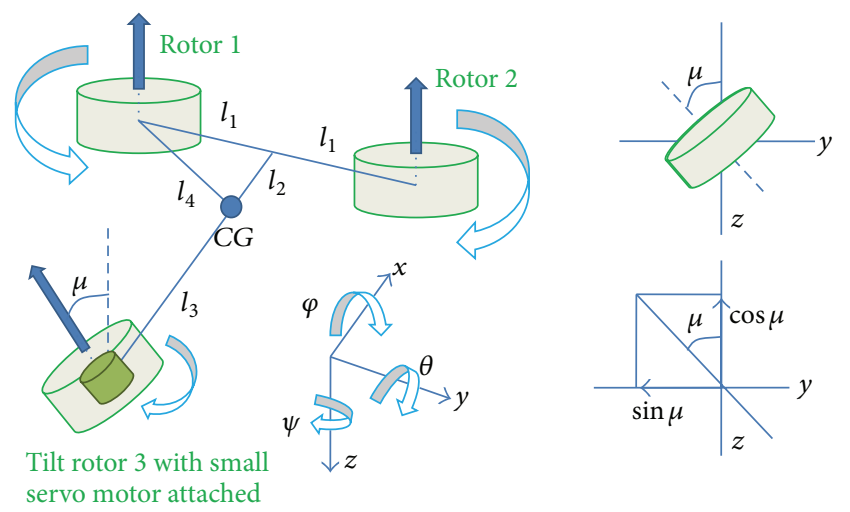

FIGURE 3: The single tilting MAV model.

manages the previous values of particle velocities in relation to the current value, along with $c_{1}$ and $c_{2}$ as learning rates. The items $\operatorname{rand}_{1}(\cdot)$ and $\operatorname{rand}_{2}(\cdot)$ are uniformly distributed random numbers between 0 and 1 . The $c_{1} \times \operatorname{rand}_{1}(\cdot) \times\left(p_{i D}(t)-x_{i D}(t)\right)$ term of (2) refers to the cognitive component, which reflects the distance at which the best solution $P_{i}(t)$ of a particle is located. The distance of particle is verified on its own.

Although evolutionary programming is similar to genetic programming, while its numerical parameters are allowed to evolve, the program structure to be optimized is fixed. The different point between EP and GA is that EP neglects crossover operator while it keeps mutation operator and selection strategy. The mutation is the main varying operator; the population members are viewed as a part of specific species rather than the same species members; therefore, each parent generates a new offspring [1].

Mutate every $x_{i}(t)$ and generate $X_{j}(t)=\left[x_{j 1}(t), x_{j 2}(t)\right.$, $\left.\ldots, x_{j D}(t)\right]$ by

$$
x_{j D}(t+1)=x_{j D}(t)+N(0,1),
$$

where $x_{j D}$ is the $j$ th individual error data and $N(0,1)$ is a normal distribution, when a random variable $x$ is distributed normally with mean of zero and one. The mutation rate is noted as mr.

The new individual is selected by using the roulette wheel technique for every particle $j$. Select $x_{j D}$ from the set of all $X_{j}$; then, update $v_{i}$ and $x_{i}$ by (2) and (3).

In this paper, by combination of PSO and EP, the proposed algorithm creates an elite HEPSO. These results usually achieve the better one than either the PSO or the existing algorithms alone.

The optimization issue is to determine a group of $\left[k_{P}, k_{I}, k_{D}, k_{e}, k_{d e}\right] \in f(E)$; therefore, the performance index of $f(E)$ is the minima. After determining the parameters of $f(E)$ for all particles, each new particle was said to represent a group of solutions.

The progression can be assigned as illustrated in Figure 2.

The fitness function integral of time multiplied by absolute error (ITAE), in this research, integrated four items such as the rise time, settling time, peak time, and maximum overshoot:

$$
\begin{aligned}
f(E)= & \alpha_{1} \times \text { Rise Time }+\alpha_{2} \times \text { Settling Time } \\
& +\alpha_{3} \times \text { Peak Time }+\alpha_{4} \times(\mid r-\text { Overshoot } \mid),
\end{aligned}
$$

where $\alpha_{i}$ are the weighting factors. The rising time, settling time, peak time, and maximum overshoot are estimated via the output performance, and then its values are recorded. Afterward, the particle groups which contain large error can be eliminated. Thus, the convergence speed of the system also accelerated. The fitness function is settled in range: $f(E) \in$ $[0,500]$.

The error behaviors of transient response and steady-state error occur at the same time, which enables the designer to offer the systematic optimization ideas and to differentiate the control system quality. Due to evaluation of the performance quality of the control system, some specific performance indicators, which combine transient response and steadystate error in the control system, are usually designed to 


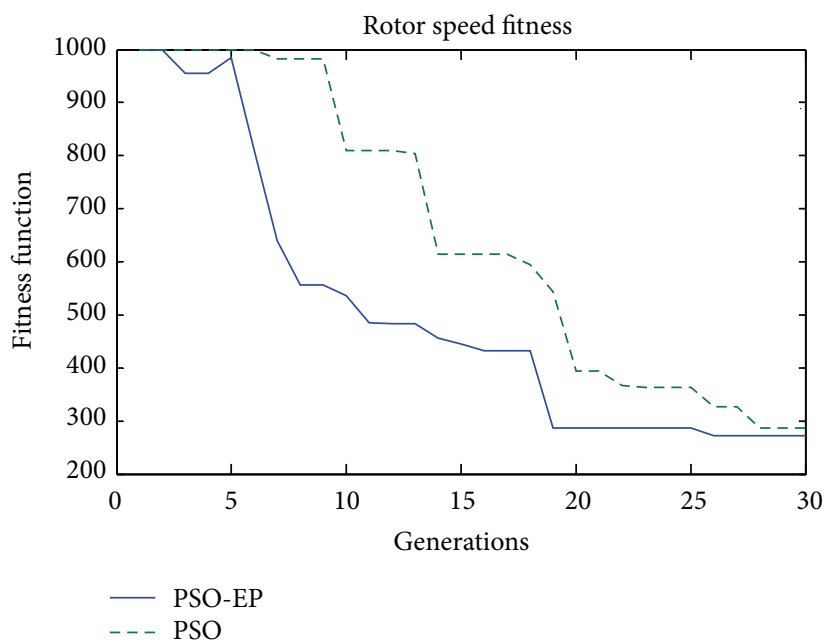

(a)

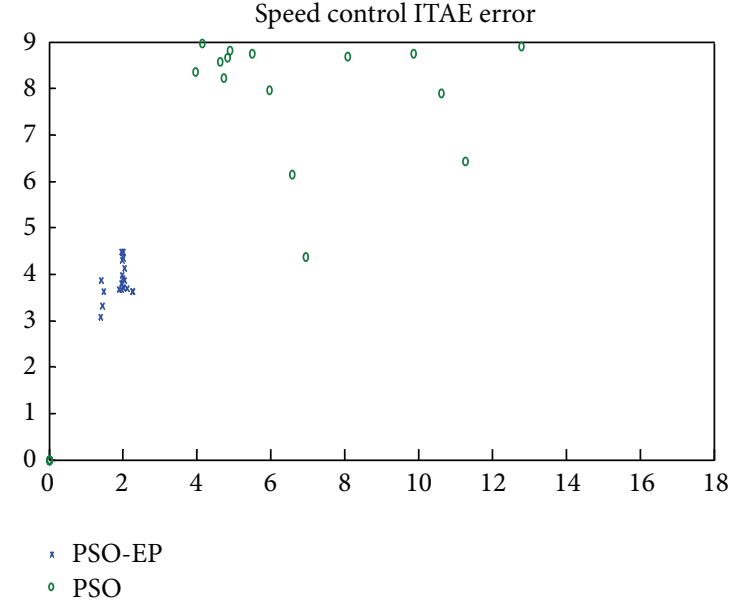

(b)

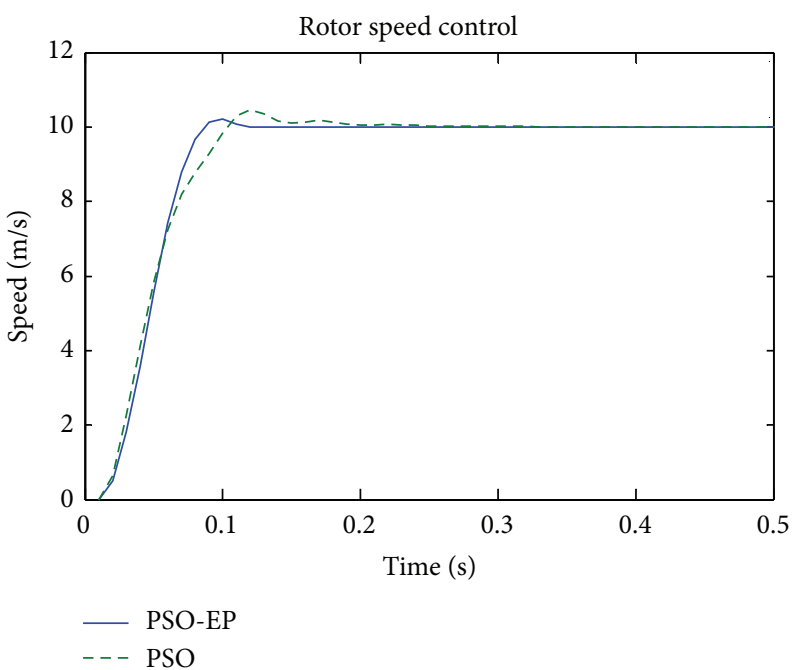

(c)

FIgURE 4: DC rotor speed control. (a) The fitness functions of the proposed controller design. (b) The convergence of ITAE error. (c) The controllers of DC rotor speed control response.

determine error criterion. The ITAE performance index [15] is mathematically given by

$$
\operatorname{ITAE}=\int_{0}^{\infty} t \cdot|e(t)| \cdot d t
$$

\section{Miniature Aerial Vehicle Model}

The MAV model is developed based on the Newton-Euler formulation. The MAV model as shown in Figure 3, which is derived from [16, 21-23], is depicted using right hand generalized earth coordinate systems of axes and body frame. The two front rotors (rotors 1 and 2) point towards positive $x$ axis, toward right rotor 2 direct $y$-axis and positive directed $z$-axis is downwards. The right handed rotation of $x-, y$-, and $z$-axis sign arrow of three positive angle variables of roll $(\varphi)$, pitch $(\theta)$, and yaw $(\psi)$, respectively. The coordinate $y$-z-axis gauges the tilt angle $\mu$.
The tilting MAV has six degrees of freedom (DOF). It has four inputs: three rotorcraft speeds and one tilt angle. The angle position is driven by one servo motor. Since two front rotors are maneuvered in different speed, the roll $(\varphi)$ control is created such as when speed of rotor 1 is down and rotor 2 is up and makes the MAV toward the left and vice versa. The third tail rotor generates the pitch $(\theta)$ control when it changes velocity. The tilting angle $\mu$ which created yaw $(\psi)$ control is driven by the small servo motor.

\section{Simulation Results}

The HEPSO algorithm parameters given by the number of population generations for speed control, servo angle position control, and altitude control are set on 30, 20, and 50 generations, respectively. The fitness weighting factor $\left[\alpha_{1}, \alpha_{2}, \alpha_{3}, \alpha_{4}\right]=[30,25,10,5]$ and PID in range are 


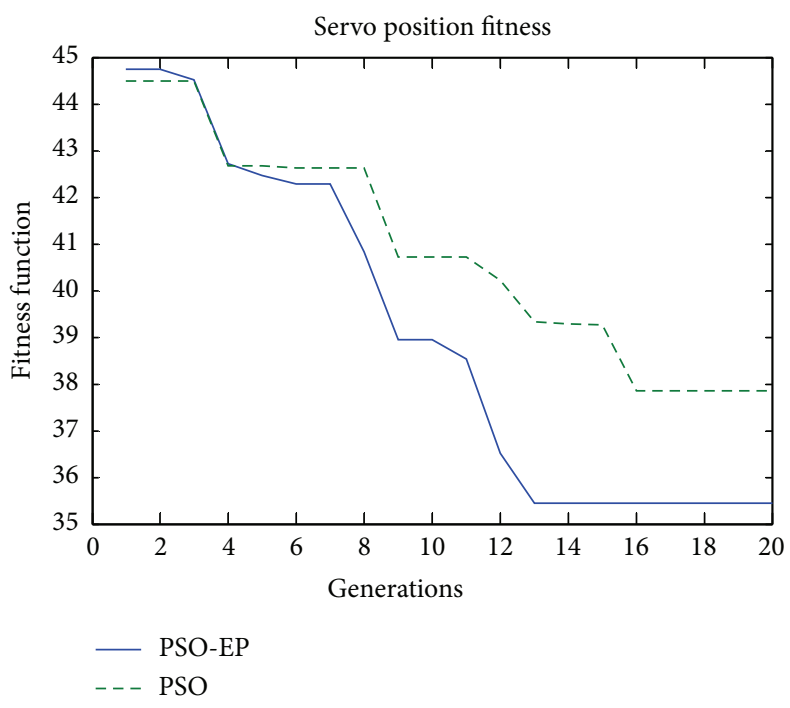

(a)

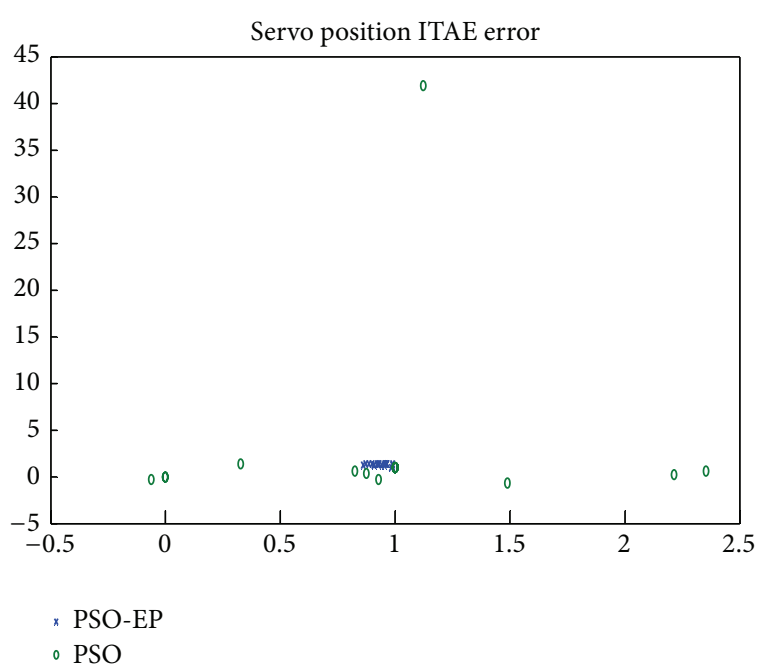

(b)

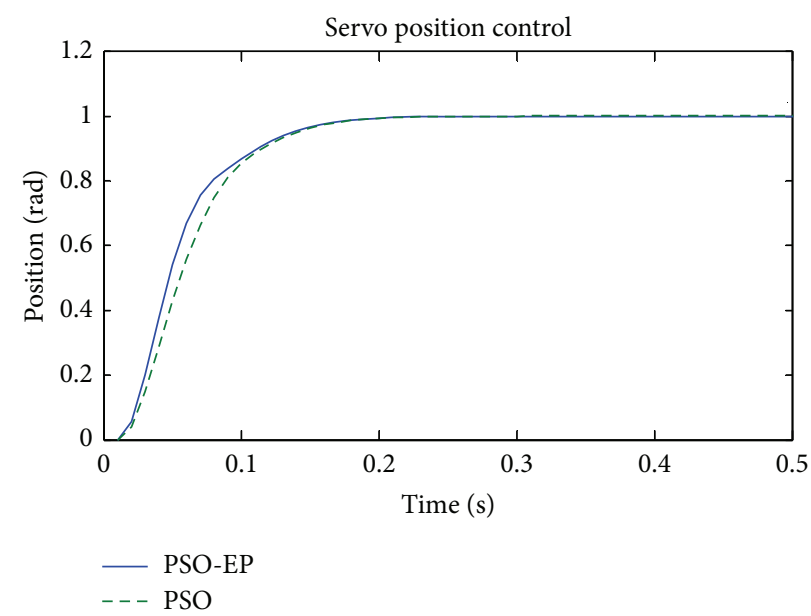

(c)

FIgURE 5: Servo motor position control. (a) The fitness functions of the proposed controller design. (b) The convergence of ITAE error. (c) The controllers of servo motor position control response.

$k_{\mathrm{PID}} \in[0,100]$. The mutation rate is $\mathrm{mr}=0.1$. The sampling time in this simulation is 0.01 .

We compared two different optimization methods which have been applied to fuzzy-PID controller gains: PSO versus HEPSO. The simulation results show that the convergence of the parameters in hybrid algorithm is yielding a higherquality solution. lows.

The transfer functions applied to controllers are as fol-

(i) Speed control (for three DC rotorcrafts): $11.4286 /(s+$ $57.14)(s+2.004)$ (Figure 4).

(ii) Servo motor position control (for the tilting angle DC servo motor with attached on the third axis): $106.7 / s(s+281.8)(s+2.582)$ (Figure 5$)$.

(iii) Altitude control (for $Z$ motion of MAV): $1 /\left(0.84 s^{2}+\right.$ $0.4286+19.376)$ (Figure 6).
The results of two controller strategies in comparison are examined: the first is PSO optimized fuzzy-PID controller and the proposed controller is HEPSO optimized algorithm aiding fuzzy-PID controller. The created generation of minimum HEPSO algorithm fitness function is faster with more than 5 generations; thus, it can save the operation time. Both of them have shown the effectiveness of the controllers design scheme.

\section{Conclusions}

In this paper, two optimization methods have been applied to MAV system models. The proposed HEPSO optimization algorithm aiding fuzzy-PID controllers have been implemented to altitude control and the MAV devices: DC motor speed control and DC servo motor position control. As obviously shown in the results, in which two controller 


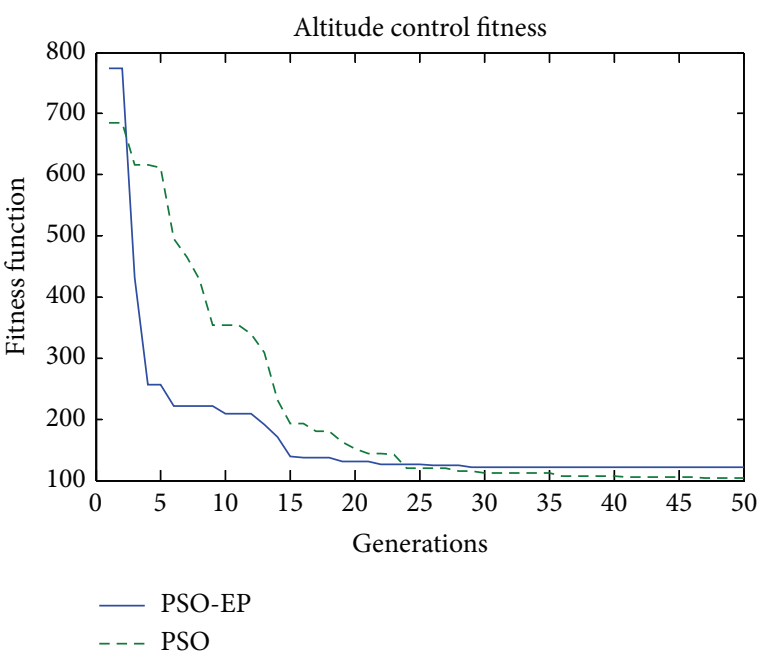

(a)

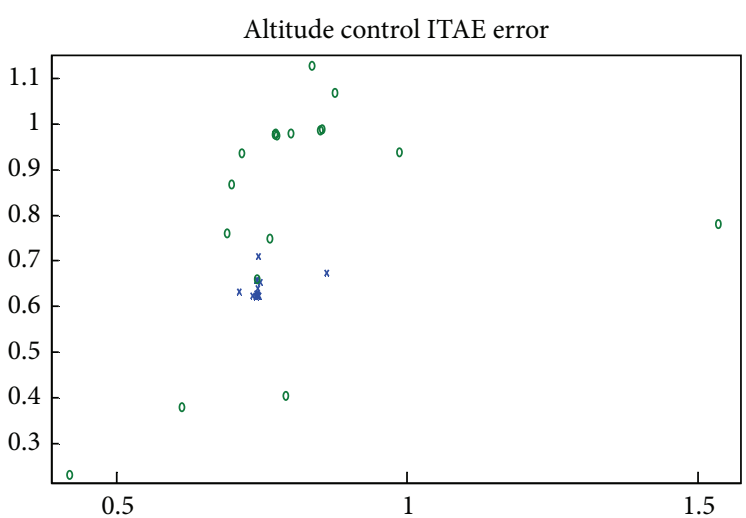

* PSO-EP
- PSO

(b)

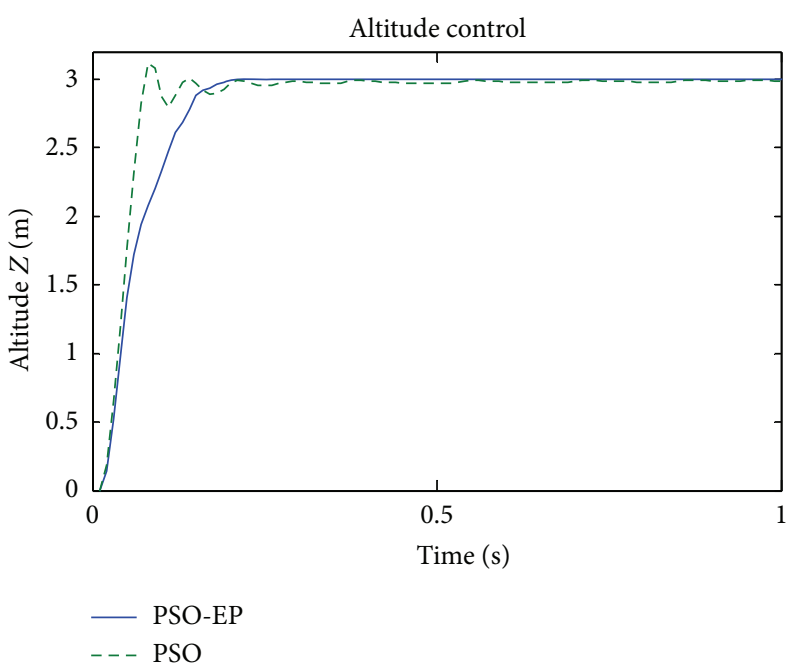

(c)

Figure 6: The altitude control. (a) The fitness functions of the proposed controller design. (b) The convergence of ITAE error. (c) The controllers of altitude control response.

configurations were tested, the most powerful proposed controllers display the best performance in terms of saving the settling time, reliability, and stability, as well. Moreover, when comparing two different optimization methods, PSO versus HEPSO, the convergence of the parameters in hybrid algorithm is yielding a higher-quality solution. The generation evolution of minimum HEPSO algorithm fitness function is faster; thus, it can save the setting and operation time. The effectiveness of the controllers design scheme for MAV is achieved.

\section{Conflict of Interests}

The authors declare that there is no conflict of interests regarding the publication of this paper.

\section{Acknowledgment}

This work is supported by the National Science Council, Taiwan, under Grant no. MOST 103-2221-E-218-027.

\section{References}

[1] A. E. Eiben and J. E. Smith, Introduction to Evolutionary Computing, Springer, New York, NY, USA, 2003.

[2] J. Kennedy and R. C. Eberhart, "Particle swarm optimization," in Proceedings of IEEE International Conference on Neural Networks, vol. 4, pp. 1942-1948, 1995.

[3] C.-F. Juang, "A hybrid of genetic algorithm and particle swarm optimization for recurrent network design," IEEE Transactions on Systems, Man, and Cybernetics B: Cybernetics, vol. 34, no. 2, pp. 997-1006, 2004. 
[4] L.-l. Li, L. Wang, and L.-h. Liu, "An effective hybrid PSOSA strategy for optimization and its application to parameter estimation," Applied Mathematics and Computation, vol. 179, no. 1, pp. 135-146, 2006.

[5] B. Niu and L. Li, "A novel PSO-DE-based hybrid algorithm for global optimization," in Advanced Intelligent Computing Theories and Applications. With Aspects of Artificial Intelligence, vol. 5227 of Lecture Notes in Computer Science, pp. 156-163, Springer, Berlin, Germany, 2008.

[6] S. Mirjalili and S. Z. M. Hashim, "A new hybrid PSOGSA algorithm for function optimization," in Proceedings of the International Conference on Computer and Information Application (ICCIA '10), pp. 374-377, Tianjin, China, November 2010.

[7] M. I. Menhas, M. R. Fei, L. Wang, and X. Fu, "A novel hybrid binary PSO algorithm," in Advances in Swarm Intelligence, vol. 6728 of Lecture Notes in Computer Science, pp. 93-100, 2011.

[8] A. Ghodrati and S. Lotfi, "A hybrid CS/PSO algorithm for global optimization," in Intelligent Information and Database Systems, vol. 7198 of Lecture Notes in Computer Science, pp. 8998, Springer, Berlin, Germany, 2012.

[9] A. A. A. Esmin and S. Matwin, "HPSOM: a hybrid particle swarm optimization algorithm with genetic mutation," International Journal of Innovative Computing, Information and Control, vol. 9, no. 5, pp. 1919-1934, 2013.

[10] Z.-Y. Zhao, M. Tomizuka, and S. Isaka, "Fuzzy gain scheduling of PID controllers," IEEE Transactions on Systems, Man and Cybernetics, vol. 23, no. 5, pp. 1392-1398, 1993.

[11] J.-S. Chiou and M.-T. Liu, "Numerical simulation for fuzzyPID controllers and helping EP reproduction with PSO hybrid algorithm," Simulation Modelling Practice and Theory, vol. 17, no. 10, pp. 1555-1565, 2009.

[12] E. N. Sanchez, H. M. Becerra, and C. M. Velez, "Combining fuzzy, PID and regulation control for an autonomous minihelicopter," Information Sciences, vol. 177, no. 10, pp. 1999-2022, 2007.

[13] R. E. Precup and S. Preitl, "PI-fuzzy controllers for integral plants to ensure robust stability," Information Sciences, vol. 177, no. 20, pp. 4410-4429, 2007.

[14] X. Guo, Y. Zhou, and D. Gong, "Optimization of fuzzy sets of fuzzy control system based on hierarchical genetic algorithms," in Proceedings of IEEE Region 10 International Conference on Electrical and Electronic Technology (TENCON '02), 2002.

[15] F. G. Martins, "Tuning PID controllers using the ITAE criterion," International Journal of Engineering Education, vol. 21, no. 5, pp. 867-873, 2005.

[16] J.-S. Chiou, H.-K. Tran, and S.-T. Peng, "Attitude control of a single tilt tri-rotor UAV SYSTEM: dynamic modeling and each channel's nonlinear controllers design," Mathematical Problems in Engineering, vol. 2013, Article ID 275905, 6 pages, 2013.

[17] J. Z. Feng, J. Li, and F. Yu, "GA-Based PID and Fuzzy logic control for active vehicle suspension system," International Journal of Automotive Technology, vol. 4, no. 4, pp. 181-191, 2003.

[18] Y.-T. Juang, Y.-T. Chang, and C.-P. Huang, "Design of fuzzy PID controllers using modified triangular membership functions," Information Sciences, vol. 178, no. 5, pp. 1325-1333, 2008.

[19] M. Clerc, "The swarm and the queen: towards a deterministic and adaptive particle swarm optimization," in Proceedings of the Congress on Evolutionary Computation (CEC '99), pp. 1951-1957, Washington, DC, USA, July 1999.

[20] R. C. Eberhart and Y. Shi, "Comparing inertia weights and constriction factors in particle swarm optimization," in Proceedings of the IEEE Congress on Evolutionary Computation, pp. 84-88, San Diego, Calif, USA, July 2000.

[21] B. L. Stevens and F. L. Lewis, Aircraft Control and Simulation, John Wiley \& Sons, New York, NY, USA, 1992.

[22] G. D. Padfield, Helicopter Flight Dynamics: The Theory and Application of Flying Qualities and Simulation Modeling, American Institute of Aeronautics and Astronautics, 1996.

[23] S. Yoon, S. J. Lee, B. Lee, C. J. Kim, Y. J. Lee, and S. Sung, “Design and flight test of a small tri-rotor unmanned vehicle with a LQR based onboard attitude control system," International Journal of Innovative Computing, Information and Control, vol. 9, no. 6, pp. 2347-2360, 2013. 


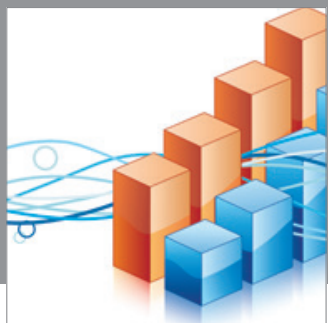

Advances in

Operations Research

mansans

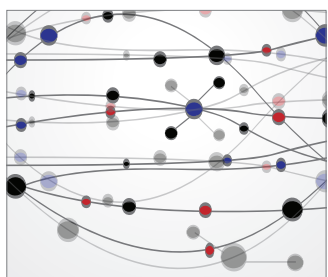

The Scientific World Journal
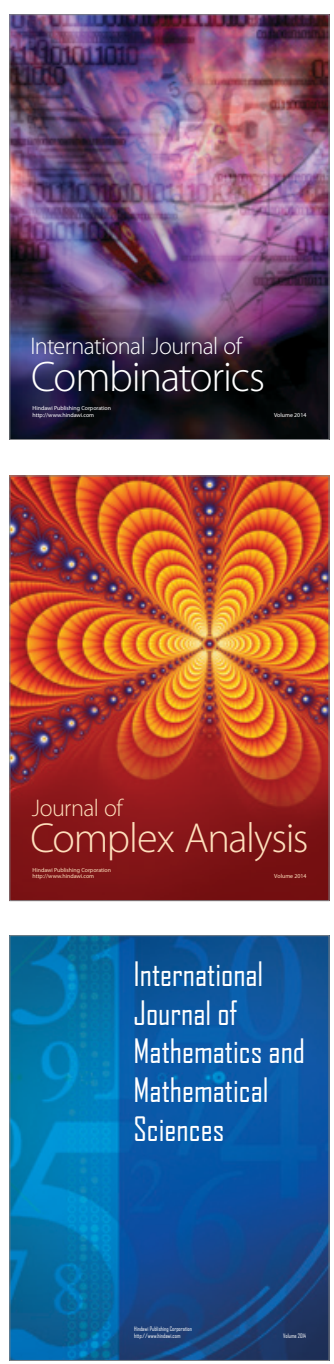
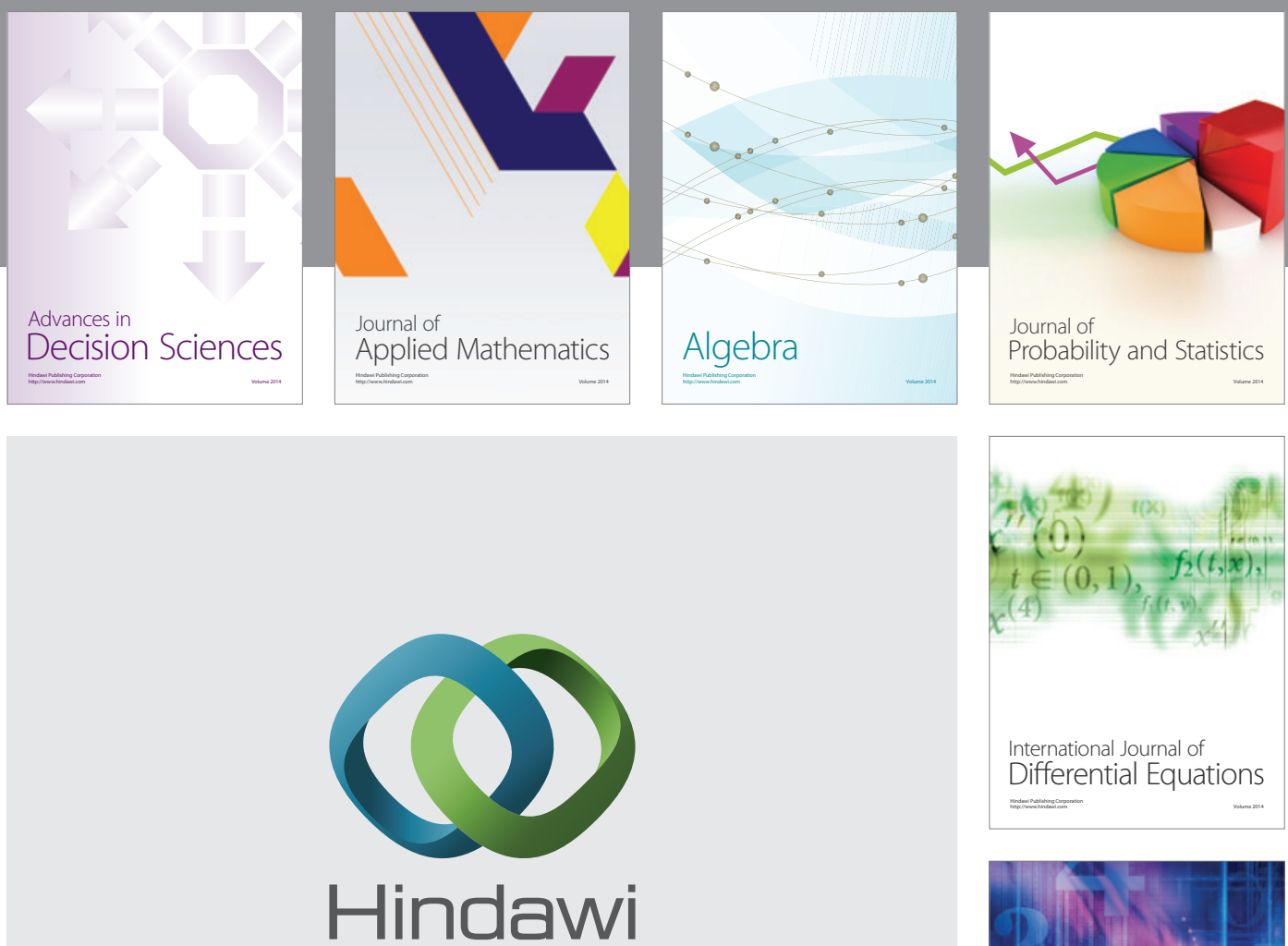

Submit your manuscripts at http://www.hindawi.com
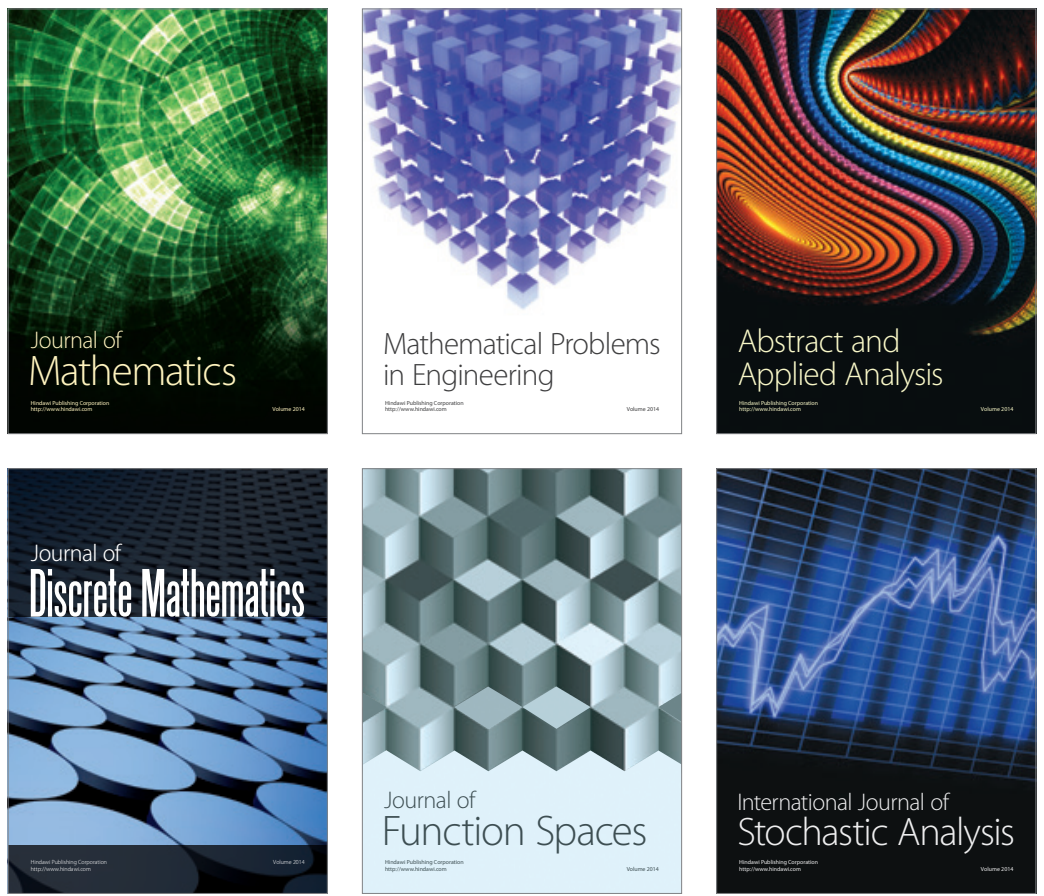

Journal of

Function Spaces

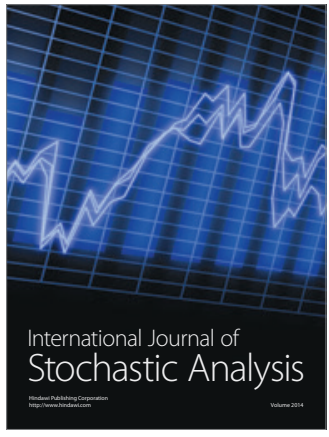

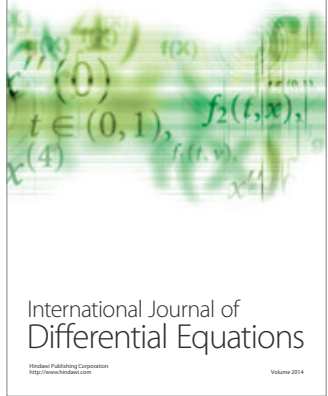
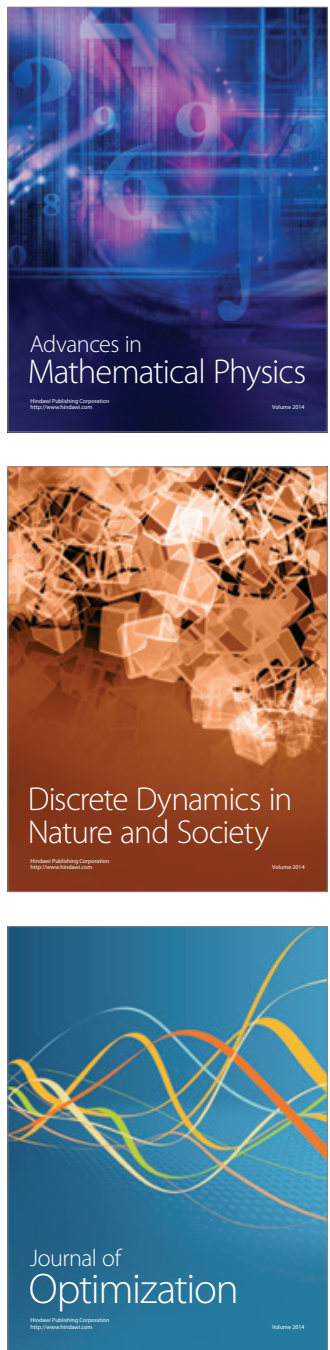